\title{
Optimal Direction Finding with Partially Calibrated Arrays
}

\author{
A. SWINDLEHURST \\ Dept. of Elec. \& Comp. Engineering \\ Brigham Young University \\ Provo, UT 8460\%
}

\begin{abstract}
This paper is concerned with the problem of optimal (maximum likelihood) direction of arrival (DOA) estimation in situations where the sensor array is calibrated over only a portion of the DOA space. Situations such as this often arise in airborne direction finding when skywave multipath is present. A parameterization is proposed for partially calibrated arrays (PCAs), and the identifiability of the model is discussed for both uncorrelated and correlated signals. It is shown how the signal and noise subspace fitting algorithms are generalized to handle PCAs, and a detection scheme is proposed for individually determining the number of signals arriving from calibrated and uncalibrated directions. The results of several simulation examples are included to validate the analysis.
\end{abstract}

\section{Introduction}

To calibrate an airborne antenna array, a single source is typically placed in a fixed location on the ground, and the aircraft flies around the source to receive data from various azimuth and depression angles. Unfortunately, this does not provide information for signals arriving from above the aircraft (unless the plane can fly upside down!), and these directions must either be ignored, or approximate calibration data (e.g., from a scale model in an anechoic chamber) substituted for them. While in most airborne direction finding (DF) applications there are no actual emitters above the collection platform, it is common for signals from groundbased emitters to reflect off upper layers of the atmosphere and be received by the aircraft from above, as depicted in Figure 1. This is especially common in the HF band, where it is referred to as the "skywave" phenomenon. Even though the array is typically on the underside of the aircraft and is primarily sensitive to signals arriving from below, a significant component of the skywave signals is coupled into the array through the aircraft frame.

There is no difficulty in implementing one-dimensional DOA estimation techniques such as MUSIC [1] in situations like those described above, one simply plots and searches the DOA spectrum only over the angles for which the array is calibrated. However, if the signals from uncalibrated DOAs are due to skywaves as in Figure 1, they can be highly correlated with the signals from calibrated directions, and one-dimensional techniques may perform poorly. Multidimensional algorithms must be used in such cases, but the question of how to parameterize a partially calibrated array (PCA) and estimate the resulting parameters becomes an important issue.

In this paper, it is shown how one might parameterize a PCA, and conditions are derived under which the resulting PCA model is identifiable. In addition, it is shown how the subspace fitting class of algorithms may be adapted to properly handle PCAs, and estimate the calibrated DOAs with minimum variance. Both the signal subspace fitting (SSF) [2] and noise subspace fitting (NSF) [3] methods will be considered. For the case of SSF, the optimal subspace weighting remains unchanged, but the form of the criterion function is modified. For NSF, the criterion remains the same, but the weighting is formed by taking a certain sub-block of the weighting in the fully calibrated case. In either case, a non-linear minimization is required only for the DOAs that arrive from calibrated angles. Since this number is

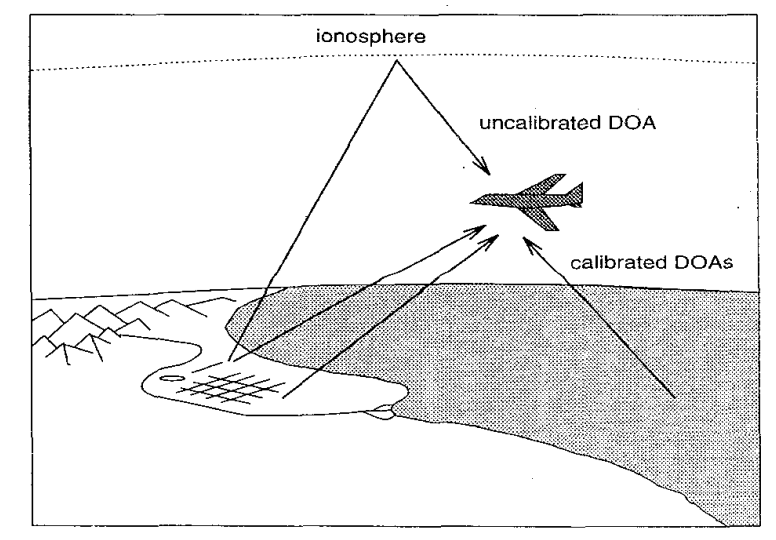

Figure 1: An airborne platform receiving direct and skywave signals. 
typically not known a priori, a technique is presented to individually estimate the number of calibrated and uncalibrated DOAs.

\section{Data Model and Relevant Algorithms}

In the standard narrowband model, the output of the array can be described by

$$
\mathbf{x}(t)=\mathbf{A}\left(\boldsymbol{\theta}_{0}\right) \mathbf{s}(t)+\mathbf{n}(t)
$$

where $\mathbf{s}(t)$ and $\mathbf{n}(t)$ are the received signal and noise, respectively, and where

$$
\mathbf{A}\left(\boldsymbol{\theta}_{0}\right)=\left[\begin{array}{lll}
\mathbf{a}\left(\theta_{1}\right) & \cdots & \mathbf{a}\left(\theta_{d}\right)
\end{array}\right] \in \mathbb{C}^{m \times d}
$$

represents the array response for $m$ sensors and $d$ total sources. If $\hat{\mathbf{E}}_{s}$ and $\hat{\mathbf{E}}_{n}$ denote the signal and noise subspace eigenvectors of the covariance of $\mathbf{x}(t)$, then the SSF and NSF (minimization) criteria can be expressed as follows:

$$
\begin{aligned}
& V_{S S F}(\boldsymbol{\theta})=\operatorname{Tr}\left(\mathbf{P}_{A}^{\perp}(\boldsymbol{\theta}) \hat{\mathbf{E}}_{s} \mathbf{W} \hat{\mathbf{E}}_{s}^{*}\right) \\
& V_{N S F}(\boldsymbol{\theta})=\operatorname{Tr}\left(\mathbf{A}^{*}(\boldsymbol{\theta}) \hat{\mathbf{E}}_{n} \hat{\mathbf{E}}_{n}^{*} \mathbf{A}(\boldsymbol{\theta}) \mathbf{U}\right),
\end{aligned}
$$

where $\mathbf{W}$ and $\mathbf{U}$ are weighting matrices, and $\mathbf{P} \frac{1}{A}(\boldsymbol{\theta})$ is the projection

$$
\mathbf{P}_{A}^{\perp}(\boldsymbol{\theta})=\mathbf{I}-\mathbf{A}(\boldsymbol{\theta})\left[\mathbf{A}^{*}(\boldsymbol{\theta}) \mathbf{A}(\boldsymbol{\theta})\right]^{-1} \mathbf{A}^{*}(\boldsymbol{\theta}) .
$$

The key feature of these algorithms is that asymptotically optimal (in the maximum likelihood sense) DOA estimates are achieved if the following weightings are applied:

$$
\begin{aligned}
\mathbf{W}_{O P T} & =\left(\hat{\boldsymbol{\Lambda}}_{s}-\hat{\sigma}^{2}\right)^{2} \hat{\boldsymbol{\Lambda}}_{s}^{-1} \\
\mathbf{U}_{O P T} & =\mathbf{A}^{\dagger}\left(\hat{\boldsymbol{\theta}}_{0}\right) \hat{\mathbf{E}}_{s} \mathbf{W}_{O P T} \hat{\mathbf{E}}_{s}^{*} \mathbf{A}^{\dagger *}\left(\hat{\boldsymbol{\theta}}_{0}\right),
\end{aligned}
$$

where $\hat{\boldsymbol{\Lambda}}_{s}$ is diagonal and contains the signal subspace eigenvalues, $\hat{\sigma}^{2}$ is a consistent estimate of the noise power, $(\cdot)^{\dagger}$ denotes a pseudo-inverse, and $\hat{\boldsymbol{\theta}}_{0}$ is a consistent estimate of the DOAs.

\subsection{PCA Model}

The above algorithms must be modified when only a PCA is available, since the array cannot always be fully parameterized by the DOAs alone. Suppose there are respectively $c$ and $u$ signals from calibrated and uncalibrated DOAs, so that $d=u+c$, and assume for simplicity that there is only one parameter per source (e.g., azimuth angle only). The case of multiple parameters (e.g., azimuth and elevation) is handled identically. Without any information about the array response in the uncalibrated directions, the array can only be parameterized as follows

$$
\mathbf{A}(\boldsymbol{\eta})=\left[\begin{array}{l:l}
\mathbf{A}(\boldsymbol{\theta}) & \mathbf{A}_{u}
\end{array}\right]
$$

where

$$
\mathbf{A}(\boldsymbol{\theta})=\left[\mathbf{a}\left(\theta_{1}\right) \cdots \mathbf{a}\left(\theta_{c}\right)\right],
$$

$\mathbf{A}_{u}$ is an $m \times u$ matrix containing the array response vectors for the uncalibrated DOAs, and

$$
\boldsymbol{\eta}=\left[\begin{array}{c}
\boldsymbol{\theta} \\
\operatorname{Re}\left\{\mathbf{A}_{u}\right\} \\
\operatorname{Im}\left\{\mathbf{A}_{u}\right\}
\end{array}\right]
$$

It will be assumed that $\mathbf{A}(\boldsymbol{\eta})$ is always full rank for signals arriving from distinct directions, and hence that the array, though only partially calibrated, is nonetheless unambiguous.

For this model, the subspace relationships that must be exploited by SSF and NSF are as follows:

$$
\begin{aligned}
& \mathbf{E}_{s}=\mathbf{A}\left(\boldsymbol{\theta}_{0}\right) \mathbf{T}_{c}+\mathbf{A}_{u} \mathbf{T}_{u} \\
& 0=\mathbf{E}_{n}^{*}\left[\begin{array}{l:l}
\mathbf{A}\left(\boldsymbol{\theta}_{0}\right) & \mathbf{A}_{u}
\end{array}\right],
\end{aligned}
$$

where $\mathbf{T}_{c}$ and $\mathbf{T}_{u}$ are $c \times d^{\prime}$ and $u \times d^{\prime}$ matrices, respectively, and where $d^{\prime} \leq d$ is the rank of the signal subspace. Thus, for the PCA problem, the array is simply parameterized by the vector $\boldsymbol{\eta}$ instead of $\boldsymbol{\theta}$ directly. As long as the PCA model is identifiable, then both the SSF and NSF estimators of $\boldsymbol{\eta}$ are guaranteed to be statistically efficient $[4,5]$. The conditions necessary for identifiability are examined below.

\subsection{Identifiability}

To begin with, note that if $\left[\mathbf{A}\left(\boldsymbol{\theta}_{0}\right): \mathbf{A}_{u}\right], \mathbf{T}_{c}$, and $\mathbf{T}_{u}$ satisfy (8)-(9), then so do $\left[\mathbf{A}\left(\boldsymbol{\theta}_{0}\right): \mathbf{A}_{u}^{\prime}\right], \mathbf{T}_{c}^{\prime}$, and $\mathbf{T}_{u}$, where

$$
\begin{aligned}
\mathbf{A}_{u}^{\prime} & =\mathbf{P}_{A}^{\perp}\left(\boldsymbol{\theta}_{0}\right) \mathbf{A}_{u} \mathbf{T}^{\prime} \\
\mathbf{T}_{c}^{\prime} & =\mathbf{T}_{c}+\mathbf{A}^{\dagger}\left(\boldsymbol{\theta}_{0}\right) \mathbf{A}_{u} \mathbf{T}^{\prime} \mathbf{T}_{u}
\end{aligned}
$$

for any full rank $u \times d^{\prime}$ matrix $\mathbf{T}^{\prime}$. Consequently, to guarantee the uniqueness of the model for $\mathbf{A}_{u}$, it is assumed that its first row is real-valued, and that $\mathbf{A}_{u}^{*} \mathbf{A}(\boldsymbol{\theta})=0, \mathbf{A}_{u}^{*} \mathbf{A}_{u}=\mathbf{I}$. The definition of $\boldsymbol{\eta}$ in (7) is thus somewhat over-simplified.

The critical issue of course is the identifiability of $\theta$ in the model above. Consider for a moment the case where there are no perfectly correlated signals $\left(d=d^{\prime}\right)$. Identifiability in $\boldsymbol{\theta}$ will be lost if there exists $\boldsymbol{\theta}^{\prime} \neq \boldsymbol{\theta}_{0}$ and a $2 d \times d$ matrix $\mathbf{P}$ such that

$$
\left[\begin{array}{l:l:l:l}
\mathbf{A}\left(\boldsymbol{\theta}_{0}\right) & \mathbf{A}_{u} & \mathbf{A}\left(\boldsymbol{\theta}^{\prime}\right) & \mathbf{A}_{u}^{\prime}
\end{array}\right] \mathbf{P}=0 .
$$

Since $\left[\mathbf{A}\left(\boldsymbol{\theta}_{0}\right) \quad \mathbf{A}_{u}\right]$ is full rank, it is clear that such a $\mathbf{P}$ will not exist if $\boldsymbol{\theta}^{\prime} \neq \boldsymbol{\theta}_{0}$ as long as $m>d$ (which 
is the standard criterion for DOA identifiability). The situation is somewhat more complicated when coherent signals are present, and space permits only a summary of the results in this case:

- The DOAs of all signals that are not coherent with signals arriving from uncalibrated directions are identifiable as long as the standard condition $m>2 d-d^{\prime}$ holds [6].

- If a signal is perfectly correlated with a signal from an uncalibrated direction, its DOA will in most cases be unidentifiable even if $m>2 d-d^{\prime}$.

An unequivocal statement in the latter case is not possible since there exist some (typically unrealistic) situations where identifiability is preserved. For example, if the signals arriving from two calibrated DOAs $\theta_{1}, \theta_{2}$ are not coherent with each other, but fully coherent with a signal from an uncalibrated DOA, then $\theta_{1}, \theta_{2}$ are identifiable.

Although there are many more parameters to be estimated with a PCA model, it will be seen in the next section that all of the uncalibrated source parameters are separable in both the SSF and NSF cost functions, and one need only search for the $c$ unknown calibrated DOAs.

\section{Detection and Estimation with PCAs}

This section presents the modifications necessary to apply the SSF and NSF algorithms to arrays parameterized by the PCA model above, assuming that both $c$ and $u$ are known. A technique for estimating $c$ and $u$ is presented at the end of the section.

\subsection{Subspace Fitting Algorithms}

Using the constraints on $\mathbf{A}_{u}$ necessary for identifiability, we have

$$
\mathbf{P}_{A}^{\perp}(\eta)=\mathbf{P}_{A}^{\perp}(\theta)-\mathbf{A}_{u} \mathbf{A}_{u}^{*} .
$$

Augmenting (2) with the constraints on $\mathbf{A}_{u}$ thus yields

$$
\begin{aligned}
V_{S S F}(\eta)= & \operatorname{Tr}\left(\mathbf{P}_{A}^{\perp}(\boldsymbol{\theta}) \hat{\mathbf{E}}_{s} \mathbf{W} \hat{\mathbf{E}}_{s}^{*}\right)+\operatorname{Tr}\left(\mathbf{A}_{u}^{*} \hat{\mathbf{E}}_{s} \mathbf{W} \hat{\mathbf{E}}_{s}^{*} \mathbf{A}_{u}\right) \\
& +\operatorname{Tr}\left(\boldsymbol{\Lambda}_{1}\left(\mathbf{A}_{u}^{*} \mathbf{A}_{u}-\mathbf{I}\right)+\boldsymbol{\Lambda}_{2} \mathbf{A}_{u}^{*} \mathbf{A}(\boldsymbol{\theta})\right)
\end{aligned}
$$

where $\boldsymbol{\Lambda}_{1}, \boldsymbol{\Lambda}_{2}$ are matrices of Lagrange multipliers. The following concentrated criterion function that depends only on the c-element vector $\theta$ can be obtained by eliminating all of the remaining separable parameters:

$$
V_{S S F}(\theta)=\sum_{i=u+1}^{d} \sigma_{i}\left(\mathbf{W}^{1 / 2} \hat{\mathbf{E}}_{s}^{*} \mathbf{P}_{A}^{\frac{1}{A}}(\theta) \hat{\mathbf{E}}_{s} \mathbf{W}^{1 / 2}\right),
$$

where $\sigma_{i}(\cdot)$ represents the $i^{\text {th }}$ singular value of its matrix argument ordered as $\sigma_{1} \geq \sigma_{2} \geq \cdots \geq \sigma_{d}$. The modified SSF criterion is thus the sum of the $c$ smallest singular values of $\mathbf{W}^{1 / 2} \hat{\mathbf{E}}_{s}^{*} \mathbf{P} \frac{1}{A}(\boldsymbol{\theta}) \hat{\mathbf{E}}_{s} \mathbf{W}^{1 / 2}$, which clearly reduces to the standard SSF criterion of (2) when $u=0$.

For NSF, the constraints on $\mathbf{A}_{u}$ yield an optimal weighting of the form

$$
\mathbf{U}_{O P T}=\left[\begin{array}{c}
\mathbf{A}^{\dagger}\left(\hat{\boldsymbol{\theta}}_{0}\right) \\
\hat{\mathbf{A}}_{u}^{*}
\end{array}\right] \hat{\mathbf{E}}_{s} \mathbf{W}_{O P T} \hat{\mathbf{E}}_{s}^{*}\left[\mathbf{A}^{\dagger *}\left(\hat{\boldsymbol{\theta}}_{0}\right) \hat{\mathbf{A}}_{u}\right]
$$

where $\hat{\mathbf{A}}_{u}$ is a consistent estimate of $\mathbf{A}_{u}$. Such an estimate can be found using a consistent estimate of $\theta_{0}$ by setting $\hat{\mathbf{A}}_{u}$ equal to the $u$ dominant singular vectors of $\mathbf{P} \frac{\perp}{A}\left(\hat{\boldsymbol{\theta}}_{0}\right) \hat{\mathbf{E}}_{s} \hat{\mathbf{E}}_{s}^{*}$. Since, given any c-element $\theta$, it is always possible to find a set of orthogonal vectors $\mathbf{A}_{u}$ that satisfy $\mathbf{A}_{u}^{*}\left[\hat{\mathbf{E}}_{n} \quad \mathbf{A}\left(\hat{\boldsymbol{\theta}}_{0}\right)\right]=0$, implementing NSF in the PCA case simply amounts to finding the $c$-element vector $\theta$ that minimizes

$$
V_{N S F}(\theta)=\operatorname{Tr}\left(\mathbf{A}^{*}(\boldsymbol{\theta}) \hat{\mathbf{E}}_{n} \hat{\mathbf{E}}_{n}^{*} \mathbf{A}(\boldsymbol{\theta}) \mathbf{U}_{c}\right),
$$

where $\mathbf{U}_{c}$ is the upper left $c \times c$ block of $\mathbf{U}_{O P T}$ in (13) As in the standard formulation of the problem, NSF does not yield consistent estimates when coherent signals are present [5], and thus SSF is preferred in such situations.

\subsection{Determining the Number of Signals}

Due to the loss of identifiability in most cases involving coherent signals, only the case where $d^{\prime}=d$ will be considered here. In such cases, an estimate of the total number of signals present is easily obtained using standard methods, but determining values for $c$ and $u$ is somewhat more difficult. Using arguments similar to those in [7], it can be shown that when $c$ and $u$ are correctly determined and $V_{S S F}$ in (12) is evaluated at its minimizing argument $\hat{\boldsymbol{\theta}}_{0}$, then $2 N V_{S S F}\left(\hat{\boldsymbol{\theta}}_{0}\right) / \hat{\sigma}^{2}$ is a $\chi^{2}$ random variable with $2 c(m-d)-c$ degrees of freedom. Using this fact, the following simple hypothesis test can be used to estimate $c$ :

1. Find an estimate $\hat{d}$ of the total number of signals using a standard detection algorithm.

2. Set $\hat{c}=\hat{d}$.

3. Let the null hypothesis be $H_{0}: c=\hat{c}$.

4. Choose a threshold $\gamma$ for the hypothesis test based on a $\chi^{2}[2 \hat{c}(m-\hat{d})-\hat{c}]$ distribution and some desired confidence region.

5. Estimate $\boldsymbol{\theta}_{0}$ under $H_{0}$, and evaluate $V_{S S F}\left(\hat{\boldsymbol{\theta}}_{0}\right)$.

6. If $2 N V_{S S F}\left(\hat{\boldsymbol{\theta}}_{0}\right) / \hat{\sigma}^{2} \leq \gamma$, accept $H_{0}$ and stop. 


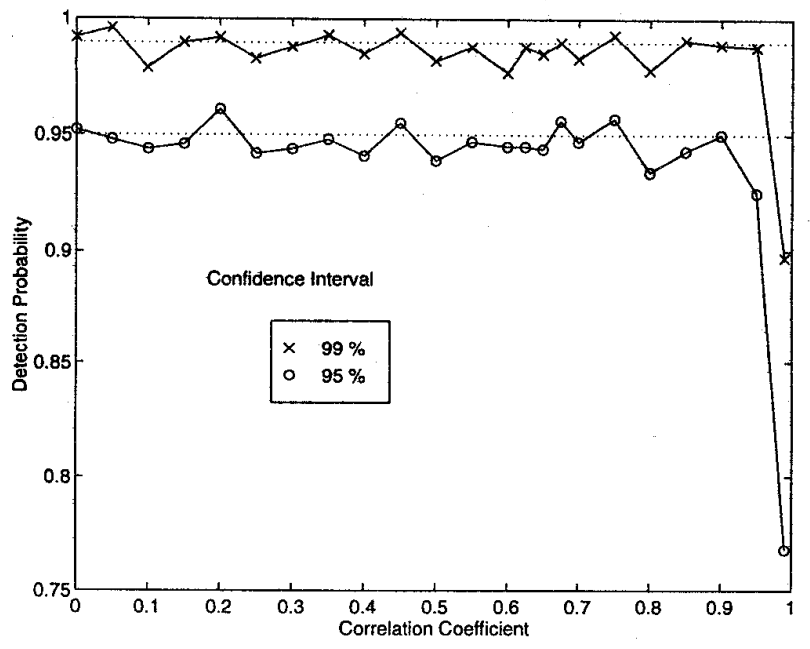

Figure 2: Probability of Correct Detection vs. Correlation Level of Uncalibrated Source

7. If $2 N V_{S S F}\left(\hat{\boldsymbol{\theta}}_{0}\right) / \hat{\sigma}^{2}>\gamma$, reject $H_{0}$ and set $\hat{c} \rightarrow \hat{c}-1$.

8. If $\hat{c}=0$, stop; otherwise, return to step 3 .

\section{A Simulation Example}

As a simple example of the techniques presented, consider a six-element uniform linear array that is "calibrated" only for angles very near broadside. Two $10 \mathrm{~dB}$ SNR signals from the calibrated DOAs $0^{\circ}$ and $5^{\circ}$ were simulated, along with a $5 \mathrm{~dB}$ SNR signal arriving from an "uncalibrated" direction of $-25^{\circ}$. The uncalibrated signal was assumed to be correlated with the broadside source with a varying correlation coefficient $\rho$. A total of 250 snapshots were used to estimate $c$ and $u$ and the calibrated DOAs, and 1000 such trials were conducted for various values of $\rho$ ranging from 0 to 0.7 . Figure 2 shows the probability of correctly determining $c=2$ for two different choices of the threshold $\gamma$ corresponding to confidence intervals of 95 and $99 \%$. In each case, the detection probabilities closely match the predicted confidence level. The RMS estimation error associated with the broadside source is plotted in Figure 3 for both MUSIC and SSF. While the performance of both algorithms degrades as correlation increases, the RMS error for SSF is significantly lower at all values of $\rho$.

\section{References}

[1] R. Schmidt, A Signal Subspace Approach to Multiple Emitter Location and Spectral Estimation, PhD thesis, Stanford University, 1981.

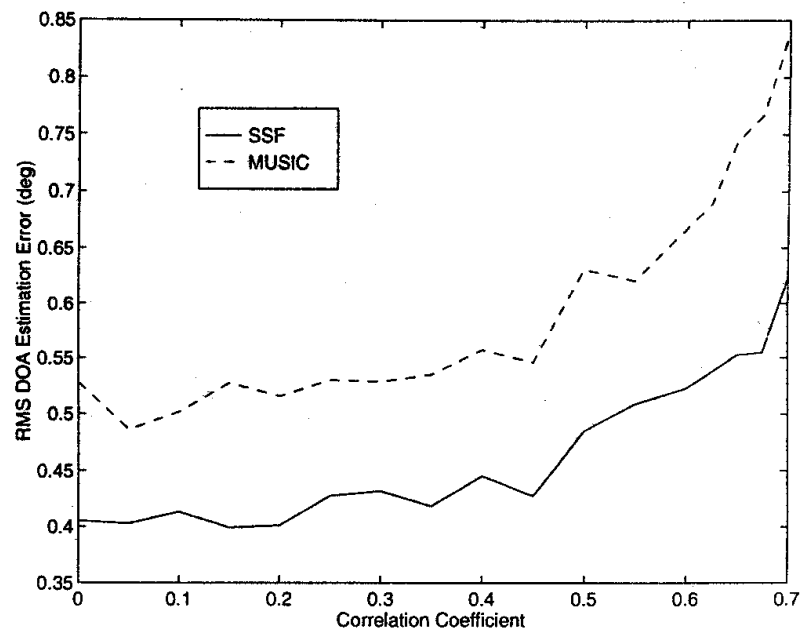

Figure 3: RMS Estimation Error of Broadside Source DOA vs. Correlation Level of Uncalibrated Source

[2] M. Viberg and B. Ottersten, "Sensor Array Processing Based on Subspace Fitting", IEEE Trans. on Sig. Proc., 39(5):1110-1121, May 1991.

[3] P. Stoica and K. Sharman, "Maximum Likelihood Methods for Direction-of-Arrival Estimation", IEEE Trans. on ASSP, 38(7):1132-1143, July 1990.

[4] B. Ottersten, M. Viberg, and T. Kailath, "Analysis of Subspace Fitting and ML Techniques for Parameter Estimation from Sensor Array Data", IEEE Trans. on Sig. Proc., 40(3):590-600, March 1992.

[5] B. Ottersten, M. Viberg, P. Stoica, and A. Nehorai, "Exact and Large Sample ML Techniques for $\mathrm{Pa}$ rameter Estimation and Detection in Array Processing", In Radar Array Processing, Haykin, Litva, and Shepherd, editors, pages 99-151. Springer-Verlag, Berlin, 1993.

[6] M. Wax and I. Ziskind, "On Unique Localization of Multiple Sources by Passive Sensor Arrays", IEEE Trans. ASSP, ASSP-37(7):996-1000, July 1989.

[7] M. Viberg, B. Ottersten, and T. Kailath, "Detection and Estimation in Sensor Arrays Using Weighted Subspace Fitting", IEEE Trans. on Sig. Proc., 39(11):2436-2449, Nov. 1991. 